\title{
FORMAL PROOF AND TEST CASE GENERATION FOR CRITICAL EMBEDDED SYSTEMS USING SCADE
}

Guy Durrieu ${ }^{2}$, Odile Laurent ${ }^{1}$, Christel Seguin ${ }^{2}$ and Virginie Wiels ${ }^{2}$

${ }^{1}$ Airbus, 316 route de Bayonne 31000 Toulouse cedex 03, France; ${ }^{2}$ ONERA/DTIM, 2 avenue Edouard Belin, BP 4025, 31055 Toulouse cedex, France

\begin{abstract}
This paper presents the work done within two research projects on the capability to use formal proof techniques for verifying properties and on the feasibility of automatic test generation for operational avionics systems.
\end{abstract}

Key words: formal techniques, verification, test generation, critical embedded systems.

\section{INTRODUCTION}

Airbus has used formal methods for several years to specify avionics systems. Thanks to these techniques, development cycles have been shortened significantly; automatic code generation from formal specification played an essential role in this improvement. A first research project conducted by Airbus and ONERA showed that the use of formal techniques for specification of avionics systems allowed the use of formal proof techniques for the validation of these systems ${ }^{1}$. Formal specifications that were studied are based on the SCADE language and a tool called SCADE Design Verifier was developed. Nowadays, SCADE Design Verifier is a commercial tool to verify properties of a formal SCADE specification and starts to be used at Airbus. Static validation techniques can thus be used in an operational way and this constitutes a first breakthrough with respect to the classical verification and validation process, which is usually based on dynamic techniques such as simulation and test. However, static analysis of 
the SCADE specifications cannot take into account all details of operational conditions and some dynamic tests such as flight tests will remain mandatory. This industrial fact and the high cost of tests definition lead us to look for assistance in the generation of test cases using automatic test generation from formal specification. This was the subject of a second research project.

This paper briefly presents the work done within these two projects (financed by DPAC, French Program Direction for Civil Aviation) on the capability to use formal proof techniques for verifying properties and on the feasibility of automatic test generation for operational avionics systems.

\section{AIRBUS SPECIFICATION WITH SCADE}

SCADE is an environment developed by Esterel Technologies (http://www.esterel-technologies.com), based on a graphical dataflow synchronous language. In this language, time is divided into discrete instants defined by a global clock. At instant $t$ the synchronous program reads inputs from external environment and computes outputs. The synchrony hypothesis states that the computation of the outputs is made at the same instant $t$. It means that for periodic systems like control/command systems, all outputs are computed at each cycle.

A SCADE specification is a set of nodes. A SCADE node is made up of constants, variables and operators. An operator may be

- a basic operator:

usual arithmetic and logical operators,

temporal operators: pre and $\rightarrow(f b y)$. Pre $(x)$ represents the value of $\mathrm{x}$ at the previous instant. Fby, called "followed by", is used to assign initial values to expressions.

- a compound operator is made up of basic operators and SCADE nodes. Within Airbus, skill oriented nodes that are very often used are called symbols. Typical symbols are: filters, triggers, integrators.

\section{CURRENT VERIFICATION AND VALIDATION MEANS}

Currently, the main verification and validation activities done at Airbus based on dynamic techniques (execution of code generated from formal specifications) are the following: 
- System level simulation: the considered system is validated in a simulated environment. The designer validates the software specification on real time computers providing a panel of commands representing possible pilot actions.

- Aircraft level simulation: several systems are validated in a simulated environment.

- System benches: first tests with real equipments, on a single system.

- Multi-systems benches: at this stage, real equipments exist for the different systems, the goal is to be sure they are correct with respect to their specification.

- General bench: last tests before the tests on the real aircraft, they are done on real equipments and with a good representation of the aircraft environment.

All the above verification and validation activities necessitate the definition of pertinent test vectors. The definition of these test cases is not easy because they have to ensure that all the functions of the system are tested and that dangerous configurations are not forgotten.

Civil airworthiness authorities require, for systems of level A, B or C, functional verification and validation activities to ensure correct operation in normal and abnormal conditions. Criteria for the termination of these functional $\mathrm{V} \& \mathrm{~V}$ activities are also to be defined. In order to meet these requirements, Airbus defines tests by identifying:

- Equivalence classes: partitioning of inputs such as a test of a given class is functionally equivalent to any other test of the same class,

- Singular points (specific behaviour),

- Limit values of inputs domains,

Structural coverage criteria, based on the structure of the specifications, chosen as termination criteria (enough tests were executed). The structural coverage criterion used is called "symbols coverage". A symbol is considered covered when a relation between inputs and outputs is set to true at least once when executing tests. This relation depends on the symbol. (Example: for a symbol A (i1:bool, i2:bool,ol:bool); a structural criterion may be the logical expression: i1 and o1). The specification is covered when all symbols coverage criteria are met.

Currently, functional test cases are described manually in test plans. The objective of our two projects was to study whether static formal techniques could improve this $\mathrm{V} \& \mathrm{~V}$ process, by replacing some test by formal verification of properties and automating part of the test generation activity. 


\section{FORMAL VERIFICATION OF PROPERTIES}

To be able to achieve formal verification, a first necessary step is the expression of properties. These properties must be derived from system requirements and formalized. The properties we considered were invariants expressing relationships between inputs and outputs of the system. They are expressed in SCADE using the synchronous observer technique.

The observed system $\mathrm{S}$ is considered as a black box and only its interface (i.e. the input and output variables) can be involved in the expression of a property $\mathrm{P}$. In order to prove that a property $\mathrm{P}$ is satisfied by a system $\mathrm{S}$ under a set of hypotheses $\mathrm{H}$, we build a system $\mathrm{S}^{\prime}$ by composition of $\mathrm{S}, \mathrm{P}, \mathrm{H}$ as shown on the figure below:

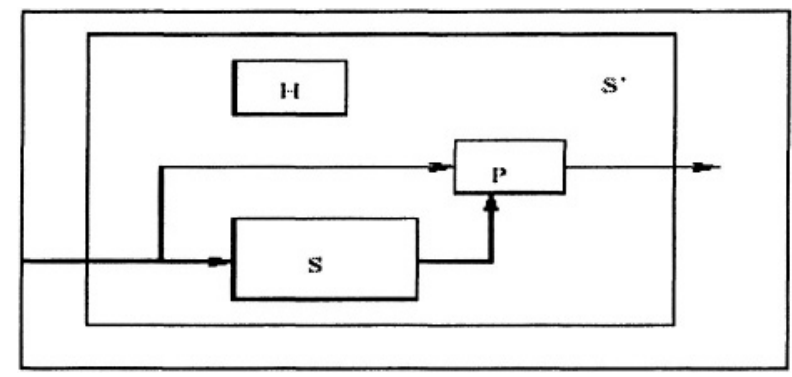

Figure 1. Obsever technique

The only output of $S^{\prime}$ is $P$. The verification then consists in checking that the output of system $S^{\prime}$ is always true.

SCADE Design Verifier is a tool developed by Prover Technologies (http://www.prover.com) based on a proof kernel. A verification can give three results:

- Valid: the property is proved.

- Falsifiable: a counter-example that falsifies the property is provided.

- Indeterminate: the tool did not succeed in proving the property that may be true or false.

We have experimented this tool on large operational systems and have had quite good results. However, we encountered difficulties with systems involving real numbers.

\section{AUTOMATIC TEST GENERATION}

As we do not want to modify the way of working of system designers, generated tests shall be defined in a very close way to the one used today at 
Airbus. So, a first issue is to find for test vectors the appropriate equivalence classes, limit values and singular points. A second issue is to evaluate the structural coverage obtained by a set of test cases. This is possible only if a pertinent structural coverage criterion is defined at the specification level. Our project thus focussed on three objectives:

Is it feasible to generate tests from a detailed specification in SCADE using formal techniques? Is it possible to identify equivalence classes?

Which coverage criterion is pertinent at the specification level?

How can a coverage analysis be implemented at the specification level?

\section{Approach}

For a given system, a set of functional test objectives is defined. Functional Test Objectives (FTO) can be defined as properties expressed in SCADE. At first, only one test case is defined for each FTO, but this is not sufficient. The behaviour class defined by the FTO has to be refined to generate several test cases for each FTO. A structural coverage criterion is then used to decide whether a sufficient refinement level has been obtained. Next paragraph describes the structural coverage criterion that has been defined for SCADE specifications. This criterion is based on the structure of the specification. It allows us to define Structural Test Objectives (STO) for each operator of the language, and a STO is a boolean condition that must be set to true at least once by a test case for the STO to be covered. Thanks to these STOs, the coverage obtained by test cases generated with respect to the FTOs can be evaluated. STOs may also be used to guide the generation of test cases or to generate new test cases if it is necessary.

\section{Structural coverage criterion}

In addition to the symbols coverage criterion already used at Airbus, we have chosen a structural coverage criterion at the specification level (adapted to SCADE specifications). The criterion has been defined by LSR-IMAG. We do not give here the detailed definition of this criterion ${ }^{2}$ but try to describe its objective in terms of coverage: for each node and for each output variable of this node, all possible values of each input variable must be exercised in a context where its value has an influence on the value of the output variable.

This criterion is very close to the constraints imposed by MC/DC (Modified Condition/Decision Coverage), the main difference is that MC/DC is defined at the code level while we are interested in a criterion at the specification level. To precisely compare both criteria, it would be necessary to study the transformations introduced by automatic code generation.

\section{TCG}

TCG (Test Case Generator) is a tool developed by Prover Technology (http://www.prover.com) based on a proof kernel. It allows to generate test 
cases for a given functional test objective while trying at the same time to cover as many structural test objectives as possible.

The tool was experimented on two main systems: the flight control secondary computer and the flight warning system. TCG has been able to generate interesting test cases and its good performances allowed us to deal with real size examples. Moreover, the tools can be parameterised to take into account the coverage criteria we have defined (symbols coverage and LSR-IMAG criterion). Nevertheless, TCG does not deliver its test cases as equivalence classes. Another tool called $\mathrm{GATEL}^{3}$ providing this essential feature was experimented, but it is not yet operational in an industrial environment.

\section{CONCLUSION AND FUTURE WORK}

The main result of both projects is to show that formal proof and automatic test generation from SCADE specification are feasible in practice. Tools exist and are able to handle real industrial examples.

Concerning automatic test generation, more work still needs to be done in two directions. Firstly, a few experimentations have been conducted to compare automatically generated tests with tests obtained with the current manual approach, but they are not sufficient. It would be interesting to fully compare both approaches by applying them in parallel on a given system with functional test objectives. Secondly, at the methodological level, the proposed approach is compliant with the certification standards. However, the proposed approach is different from the classical coverage analysis at the code level, so we think it is necessary to study more thoroughly the possible substitution of the coverage analysis at the code level by a coverage analysis at the specification level. This reflection should be part of a more global study of the integration of this approach to the actual verification and validation process. This global study should also address the complementarity of proof and test generation techniques.

\section{REFERENCES}

1. O. Laurent, P. Michel, V. Wiels. Using formal verification techniques to reduce simulation and test effort. FME 2001. LNCS 2021, Jose Nuno Oliveira and Pamela Zave eds. Springer Verlag.

2. A. Lakehal, I. Parissis, L. du Bousquet. Critères de couverture structurelle de programmes Lustre. Proceedings of AFADL 04.

3. B. Marre, A. Arnould. Test Sequence Generation from Lustre Descriptions: GATEL. $15^{\text {th }}$ international conference on Automated Software Engineering (ASE) 2000. 